\title{
The Montreal Protocol for Identification of Amusia
}

\author{
D. T. Vuvan ${ }^{1,2} \cdot$ S. Paquette ${ }^{2,3}$ - G. Mignault Goulet ${ }^{2,4} \cdot$ I. Royal $^{2,4} \cdot$ M. Felezeu ${ }^{2,4}$ • \\ I. Peretz $z^{2,4}$
}

Published online: 28 April 2017

(C) Psychonomic Society, Inc. 2017

\begin{abstract}
The Montreal Battery for the Evaluation of Amusia (MBEA; Peretz, Champod, \& Hyde Annals of the New York Academy of Sciences, 999, 58-75, 2003) is an empirically grounded quantitative tool that is widely used to identify individuals with congenital amusia. The use of such a standardized measure ensures that the individuals tested will conform to a specific neuropsychological profile, allowing for comparisons across studies and research groups. Recently, a number of researchers have published credible critiques of the usefulness of the MBEA as a diagnostic tool for amusia. Here we argue that the MBEA and its online counterpart, the AMUSIA tests (Peretz et al. Music Perception, 25, 331-343, 2008), should be considered steps in a screening process for amusia, rather than standalone diagnostic tools. The goal of this article is to present, in detailed and easily replicable format, the full protocol through which congenital amusics should be identified. In providing information that has often gone unreported in published articles, we aim to clarify the strengths and limitations of the MBEA and to make recommendations for its
\end{abstract}

The original version of this article was revised: On page 4, right-hand column, under Melodic organization tests, the first heading should be changed from "The contour test" to "The scale test".

\section{T. Vuvan}

d.vuvan@gmail.com

1 Psychology Department, Skidmore College, Saratoga Springs, NY, USA

2 International Laboratory for Brain, Music, and Sound Research, Montreal, Quebec, Canada

3 Beth Israel Deaconess Medical Center, Harvard Medical School, Boston, MA, USA

4 Department of Psychology, University of Montreal, Montreal, Quebec, Canada continued use by the research community as part of the Montreal Protocol for Identification of Amusia.

Keywords Amusia $\cdot$ MBEA $\cdot$ Accuracy $\cdot d$-Prime $\cdot$ Standardized protocol $\cdot$ Diagnosis

A challenge of neuropsychological research on special populations, such as congenital amusia, is the need to standardize the methods used to identify members of these populations. The use of standardized methods ensures that individuals tested for this research conform to the appropriate neuropsychological profile, such that conclusions from these studies will be internally valid, as well as externally comparable to findings from other studies of the same population. Congenital amusia is a neurodevelopmental disorder of pitch perception that cannot be explained by hearing loss, brain damage, intellectual deficits, or lack of music exposure. In the 14 years since Peretz and colleagues published the Montreal Battery for the Evaluation of Amusia (MBEA; Peretz, Champod, \& Hyde, 2003), research on congenital amusia has exploded, with this original article being cited 523 times since its publication. The MBEA, based on a modular view of musical pitch processing (Peretz \& Coltheart, 2003), provided researchers with an empirically grounded quantitative tool to identify individuals with congenital amusia. This was followed a few years later by a collection of easy-to-use online AMUSIA tests (Peretz et al., 2008). This explosion of research is no surprise, because this disorder of musical pitch processing offers a natural experiment with which to ask questions about brain development and plasticity (e.g., Mignault Goulet, Moreau, Robitaille, \& Peretz, 2012), modular processing between music and language (e.g., Vuvan, Nunes-Silva, \& Peretz, 2015), auditory-evoked emotion perception (e.g., Gosselin, Paquette, \& Peretz, 2015; Marin, Thompson, Gingras, \& Stewart, 2015; 
Thompson, Marin, \& Stewart, 2012), the neurocognitive underpinnings of perception and action (e.g., Dalla Bella, Giguère, \& Peretz, 2009; Hutchins \& Peretz, 2012; Royal, Lidji, Théoret, Russo, \& Peretz, 2015), and gene-environment interactions (e.g., Peretz, Cummings, \& Dubé, 2007).

However, the utility of the MBEA to effectively identify individuals with congenital amusia (amusics, henceforth) has been called into question. Three articles have provided a critical perspective on the use of the MBEA as a diagnostic tool (Henry \& McAuley, 2010, 2012; Pfeifer \& Hamann, 2015). These authors have argued that the MBEA is a suboptimal diagnostic tool for three reasons. First, scores on the MBEA (both global and for the constituent tests) form a negatively skewed distribution. Typically, individuals who obtain a global score lower than two standard deviations below the mean are classified as amusics (with a statistical prevalence of 2.5\%). Both Henry and McAuley (2010; 2012) and Pfeifer and Hamann (2015) rightly observed that the use of this type of cutoff on a negatively skewed distribution leads to an overdiagnosis of amusic cases. Pfeifer and Hamann (2015) additionally reported a problem of underdiagnosis in some cases, particularly when considering the nonmelodic subtests of the MBEA. These authors suggest remedying this problem by using $d$-prime rather than accuracy to quantify MBEA scores. A second concern is that different articles, as well as different research groups, have identified amusics by using varying cutoff scores (Table 1). For instance, some researchers have moved to using a cutoff that uses the score on only the melodic tests of the MBEA, given evidence that amusia is specific to pitch processing. Other researchers, however, have moved away from using a specific cutoff, and instead have recruited a control group whose scores are compared statistically to those from the amusic group. A final concern introduced by Pfeifer and Hamann (2015) is that web-based evaluation is not as reliable as laboratory evaluation.

These three concerns are empirically substantiated and deserve serious consideration. The crux of the critiques is that the MBEA is a suboptimal tool for the diagnosis of amusia, and there is credible evidence for this claim. However, we argue that the MBEA and its online counterpart, the AMUSIA tests, should not be considered the sole tools with which to identify amusics. Rather, use of the MBEA is just a single step in an extended process, taking place both online and in the lab, for identifying amusic participants for scientific study. The goal of this article is to present, in detailed and easily replicable format, the full protocol with which congenital amusics are identified in our laboratory: the Montreal Protocol for Identification of Amusia (MPIA). By providing information that often goes unreported in published articles, we aim to clarify the strengths and limitations of the MBEA and to make recommendations for its continued use by the research community.

\section{Screening versus diagnosis}

To put our methods in context, it is important to explore the conceptual distinction between the terms diagnosis and screening. Screening is defined as the examination of a large number of subjects for the detection of characteristics of interest. Screening tests are part of a clinical approach and allow the identification of clinical signs (such as poor pitch perception) that suggest the need for further evaluation, but they are not alone sufficient to establish a diagnosis. The online AMUSIA tests can be considered one such screening tool for the identification of amusic individuals. In contrast, diagnosis is the investigative process of assigning a condition label to explain the clinical signs observed in an individual.

Diagnosis requires the use of multiple investigative tools that assess the characteristics of interest (e.g., the MBEA) but also allow for the ruling out of other conditions that can explain the clinical signs observed. This is why the participants in our studies are subjected to a number of audiometric tests, cognitive assessments, and questionnaires in addition to the MBEA. That being said, it is important to recognize that the act of making an official diagnosis is reserved for certain clinical professions. In our laboratory, the aim is never to make a clinical diagnosis per se, but rather to identify individuals who have a profile compatible with congenital amusia, in order to study these abnormalities. To recapitulate, the MBEA and the web-based screening tool AMUSIA are both neuropsychologically grounded investigative tools that constitute important parts of the protocol we use to identify amusic participants for our research.

The characterization of the MBEA as an investigative screening tool rather than a standalone diagnosis has important implications for the present three critiques of its use. First, in cases in which the use of accuracy scores with the MBEA tends to be liberal (i.e., when it "overdiagnoses" amusia), this is a strength for identifying amusics, because researchers will be less likely to erroneously discard amusic participants at early stages of the identification process. Participants who are identified by the MBEA as potentially amusic, but who in fact are not amusic, will then be filtered out by the assessments that follow. Researchers trying to identify amusics for study are less affected when the use of accuracy scores with the MBEA is too conservative (i.e., "underdiagnoses" amusia), since missing a potential amusic case does not contaminate the amusic sample for a particular study. Second, the use of different cutoffs by different groups should be construed as a feature, rather than a weakness, of the MBEA. For instance, in recent years researchers have classified a new musical deficit called beat deafness (Phillips-Silver et al., 2011). Beat deafness is a deficit in the processing of musical time and is independent of the pitch-based congenital amusia on which we focus in the present article. The existence of two dissociable musical deficits is in accordance with the 
Table 1 Amusia identification methods for studies using the MBEA (2004-2013; drawn from a meta-analysis by Vuvan et al., 2015)

\begin{tabular}{|c|c|c|c|}
\hline Author & Year & MBEA Component & Categorization Method \\
\hline Foxton, Dean, Gee, Peretz, \& Griffiths & 2004 & Global & Cutoff \\
\hline Hyde \& Peretz & 2004 & Melodic & Cutoff \\
\hline Hyde \& Peretz & 2005 & Global & Comparison \\
\hline Loui, Guenther, Mathys, \& Schlaug & 2008 & Melodic & Cutoff \\
\hline Patel, Wong, Foxton, Lochy, \& Peretz & 2008 & Global & Cutoff \\
\hline Peretz et al. & 2008 & Global & Cutoff \\
\hline Gosselin, Jolicœur, \& Peretz & 2009 & Global & Cutoff \\
\hline Loui, Alsop, \& Schlaug & 2009 & Contour & Cutoff \\
\hline Peretz et al. & 2009 & Global \& melodic & Comparison \\
\hline Tillmann, Schulze, \& Foxton & 2009 & Global & Cutoff \\
\hline Hutchins et al. & 2010 & Global & Comparison \\
\hline Hutchins, Gosselin, \& Peretz & 2010 & Global & Cutoff \\
\hline Jiang, Hamm, Lim, Kirk, \& Yang & 2010 & Global & Cutoff \\
\hline Liu, Patel, Fourcin, \& Stewart & 2010 & Melodic & Cutoff \\
\hline Nan, Sun, \& Peretz & 2010 & Global & Comparison \\
\hline Pfeuty \& Peretz & 2010 & Each subtest individually & Comparison \\
\hline Tremblay-Champoux, Dalla Bella, Phillips-Silver, Lebrun, \& Peretz & 2010 & Global & Cutoff \\
\hline Williamson \& Stewart & 2010 & Melodic & Cutoff \\
\hline Williamson, McDonald, Deutsch, Griffiths, \& Stewart & 2010 & Melodic & Cutoff \\
\hline Jiang, Hamm, Lim, Kirk, \& Yang & 2011 & Global & Cutoff \\
\hline Omigie \& Stewart & 2011 & Melodic & Cutoff \\
\hline Tillmann, Burnham, et al. & 2011 & Global & Cutoff \\
\hline Tillmann, Rusconi, et al. & 2011 & Global & Cutoff \\
\hline Williamson, Cocchini, \& Stewart & 2011 & Melodic & Cutoff \\
\hline Anderson, Himonides, Wise, Welch, \& Stewart & 2012 & Melodic & Comparison \\
\hline Hutchins \& Peretz & 2012 & Global & Comparison \\
\hline Jiang et al. & 2012 & Global \& melodic & Comparison \\
\hline Liu, Jiang, et al. & 2012 & Melodic & Cutoff \\
\hline Liu, Xu, Patel, Francart, \& Jiang & 2012 & Each subtest individually & Comparison \\
\hline Loui \& Schlaug & 2012 & Melodic & Comparison \\
\hline Marin, Gingras, \& Stewart & 2012 & Melodic & Cutoff \\
\hline Mignault Goulet et al. & 2012 & Scale & Cutoff \\
\hline Omigie, Pearce, \& Stewart & 2012 & Melodic & Cutoff \\
\hline Thompson, Marin, \& Stewart & 2012 & Melodic & Cutoff \\
\hline Williamson, Liu, Peryer, Grierson, \& Stewart & 2012 & Melodic & Cutoff \\
\hline Albouy, Mattout, et al. & 2013 & Global & Cutoff \\
\hline Albouy, Schulze, Caclin, \& Tillmann & 2013 & Global & Cutoff \\
\hline Hutchins \& Peretz & 2013 & Global & Comparison \\
\hline Jiang, Lim, Wang, \& Hamm & 2013 & Each subtest individually & Comparison \\
\hline Moreau, Jolicoeur, \& Peretz & 2013 & Global & Comparison \\
\hline
\end{tabular}

In all cases using the cutoff method, cutoffs were drawn from Peretz et al. (2003). The specific cutoff values were as follows: Global, 23/30; Melodic, 65/ 90; Scale, 22/30; Contour, 22/30; Interval, 21/30; Rhythm, 23/30; Meter, 20/30; Memory, 22/30. In all cases using the comparison method, the amusic group's MBEA scores were shown to be significantly different from a matched control group's MBEA scores.

modular view of music processing that drove the development of the MBEA (Peretz \& Coltheart, 2003). That these two disorders can be dissociated by using MBEA subtest cutoffs rather than the traditional full-scale cutoffs is a strength of the structure of this protocol. In fact, the modular nature of the
MBEA provides built-in control assessments (meter and memory subtests act as controls for the identification of pitch-based amusia, and melodic and memory subtests act as controls for the identification of beat deafness). Finally, the unreliability of the web-based AMUSIA test as compared to 
its laboratory-based counterpart is addressed in our protocol by using the online test as the initial screening step, which is followed up in the laboratory by the full-scale MBEA and by multiple additional assessments, as will be revealed in the material that follows.

\section{The Montreal Protocol for Identification of Amusia (MPIA)}

\section{Web-based screening (30 $\mathrm{min}$ total)}

\section{AMUSIA online screening tests $(30 \mathrm{~min})$}

Since their original publication (Peretz et al., 2008), the AMUSIA online screening tests have been updated (Peretz \& Vuvan, 2017). This screening tool is composed of three tests and a questionnaire. Each of the three tests is preceded by multiple examples, to aid participants in task comprehension. Unpublished analyses of AMUSIA data have indicated that the out-of-tune test (part of the originally published online test; Peretz et al., 2008) shows low construct validity (i.e., does not correlate significantly with the melodic subtests of the MBEA) and is highly correlated with the out-of-key test. Thus, the out-of-tune test has been replaced in this version by the scale test from the MBEA (described below), which has been used by many researchers to identify amusia (see Table 1).

The scale test is excerpted from the laboratory-based MBEA (see below). Participants are presented with 31 pairs of melodies (of which one is a catch trial). Half of the melody pairs are identical, and half contain one melody with a single changed note that is in tune but out of scale. Participants are asked simply to judge whether the two melodies are the same or different.

The off-beat test contains 24 melodies, half of which are manipulated to contain a delay that causes the rest of the melody to be off the beat. Participants must judge whether each melody contains a temporal incongruity.

The out-of-key test contains 24 melodies, half of which are manipulated to contain one note that is out of key. Participants must judge whether each melody contains a pitch incongruity.

The questionnaire contains 32 questions regarding participants' education and professional backgrounds, everyday musical habits, musical ear, childhood musical experience, musical education, and health and hearing.

Participants are identified as potential amusics if they score less than $70 \%$ on both the scale test and the out-of-key test, score above $70 \%$ on the off-beat test, and respond positively to at least one of the following statements: (1) "I cannot recognize familiar tunes without the help of the lyrics," (2) "I cannot tell if I sing in tune," and (3) "I have been told that I sing out-of-tune." Participants are excluded if they report any traumatic brain injury. Note that these cutoffs are very liberal, in keeping with our goal of using the AMUSIA tests as a screening tool. These potential amusic participants are invited to come into the laboratory for further evaluation.

\section{Laboratory evaluation (2 h 15 min total)}

The evaluation of potential amusics in the laboratory consists of five tasks.

\section{Audiometry (10 min)}

Audiometric thresholds are measured to ensure that poor performance on any of the following five tests is not due to hearing impairments. Individuals whose hearing must be corrected, or those with hearing thresholds outside of the norms for their age group (as determined by the ISO-1999 standards) are excluded from consideration as amusics.

\section{MBEA (60 min)}

This assessment is composed of six tests that assess the functioning of each of the musical processing components described by Peretz and Coltheart (2003). Each test consists of 30 trials and is preceded by examples (four examples for the metric test and two examples for all other tests). In addition to these experimental trials, one catch trial (Trial 31) in each of the tests requires the comparison of two sequences (scale, contour, interval, or rhythm).

Melodic organization tests All tests of melodic organization present pairs of melodies that participants must judge to be identical or different.

The scale test In half the trials, one of the melodies contains a critical pitch that is altered to be out of scale, without changing the overall contour of the melody. Note that this test (which forms part of the AMUSIA online screening) is reassessed in the lab as part of the MBEA because of concerns regarding the reliability of Web testing (e.g., Pfeifer \& Hamann, 2015).

The contour test In half the trials, one of the melodies contains a critical pitch that is altered to change the contour (pitch direction of the surrounding intervals), without violating the scale of the melody.

The interval test In half the trials, one of the melodies contains a critical pitch that is altered, without changing the scale or contour of the melody. 


\section{Temporal organization tests}

The rhythm test In this test, pairs of melodies are presented, which participants must judge to be identical or different. In half the trials, the rhythmic grouping of one of the melodies is altered by changing the durations of two adjacent tones, thus altering their temporal proximity. The meter and total number of sounds are unaltered.

The meter test In this test, listeners hear two-phrase harmonized sequences (twice as long as the onephrase melodies used in the previous four MBEA subtests) and must judge whether the sequences are marches (duple meter) or waltzes (triple meter).

Memory recognition test From the original set of 30 melodies used in the preceding tests, 15 appear in the recognition test, as well as an additional 15 novel foil melodies. On each trial a melody is presented, and participants must judge whether or not they had heard the melody during the previous tests.

Since researchers have come to a consensus regarding the specificity of pitch to amusia (i.e., in contrast to beat deafness; see Peretz, 2016; Vuvan et al., 2015), participants must generally obtain a melodic score (combination of all the melodic organization tests) below the statistical cutoff. A discussion of how a statistical cutoff might be obtained appears in the Calculating Cutoffs section. It might be expected that pitch-based amusics would perform normally on the temporal and memory subtests as well. However, amusic performance on these "control" subtests tends to be heterogeneous, partly because the mere presence of pitch in these subtests interferes with some individuals' performance (Phillips-Silver, Toiviainen, Gosselin, \& Peretz, 2013). Thus, we use additional tests as cognitive-control tasks (see the following section).

\section{Cognitive deficit screening (30 $\mathrm{min})$}

Two tasks from the Wechsler Adult Intelligence Scale (WAISIII; Wechsler, 1997) are employed to exclude individuals who perform poorly on the MBEA for reasons that are unrelated to amusia.

Matrix reasoning assesses logical reasoning and problem solving and is correlated with full-scale IQ (Tulsky, Zhu, \& Ledbetter, 1997). This test ensures that an MBEA score below the cutoff is not due to intellectual impairments.

Digit span assesses auditory attention, as well as short-term and working memory. All of the tests included in the MBEA, except for the meter test, impose a working memory load by asking participants to compare two excerpts of music that are presented in sequence. The digit span test ensures that an MBEA score below the cutoff is not due to the impairment of working or short-term memory.

Individuals with matrix reasoning or digit span scaled scores lower than 6 (i.e., the 9 th percentile) are excluded from consideration as amusics.

\section{Pitch discrimination (25 min)}

The ability to make fine-grained discriminations between pitches is assessed using a pitch change detection task. During this task, participants hear five-tone sequences and must judge whether the fourth tone differed in pitch from the others (similar to the task from Hyde \& Peretz, 2004). A total of 360 sequences are presented, with half of these sequences containing a pitch change on the fourth tone, equally distributed in terms of size (quarter semitone, half semitone, one semitone, two semitones, three semitones) and direction (up and down).

Pitch discrimination performance is not used strictly to identify amusics, since cases do exist in which it would be interesting to study individuals who fail at music perception (measured with the MBEA), despite normal pitch discrimination. Nevertheless, since amusia is generally understood to be a disorder of fine-grained pitch perception, amusics generally have pitch change detection thresholds close to one semitone (the smallest meaningful pitch distance in Western music; Hyde \& Peretz, 2004).

\section{Pitch production (10 min)}

Singing abilities are tested using a protocol developed by Dalla Bella et al. (2009). In this test, participants are recorded while singing a familiar tune ("Gens du Pays" by Gilles Vigneault) with familiar lyrics (the birthday song "Mon Cher Michel"). If the participant is not familiar with this song, he or she is asked to sing "Happy Birthday" (in English) or "Bonne Fête" (in French). The song is sung once with the words and once on the syllable "la" in four different conditions: (1) singing alone, (2) singing along with a model, (3) singing alone following the model's example, and (4) singing along with a metronome.

Singing performance is not used strictly to identify amusics, since previous research has shown a dissociation between pitch perception and production in a subset of amusics (Dalla Bella et al., 2009; Hutchins, Zarate, Zatorre, \& Peretz, 2010). However, amusics do generally show poor performance (as quantified by the degree and number of pitch errors) on this singing task. 


\section{Summary and notes}

To summarize, to be considered amusic, an individual must have normal hearing (age-standardized, no hearing correction), no history of traumatic brain injury, and perform within the normal range on cognitive tests that screen for attention and intellectual difficulties, and must also obtain an MBEA melodic score below the cutoff, without failing any catch trials (see the next section). Note that it is not necessary to use the specific WAIS-III tasks described here to screen for nonmusical cognitive impairments, as long as some form of screening is applied. One alternative possibility is the Montreal Cognitive Assessment (Nasreddine et al., 2005; mocatest.org), a brief cognitive-screening tool that is free to use.

Additional information on pitch change detection, singing performance, and musical background may be leveraged, depending on the specific research questions being asked. In particular, some researchers have used pitch discrimination thresholds in conjunction with or instead of the MBEA to identify amusics (e.g., Loui, Alsop, \& Schlaug, 2009). A scoring sheet for the MPIA can be found at www.peretzlab.ca/ knowledge_transfer/.

\section{Calculating cutoffs: Theoretical and empirical considerations}

As we alluded to previously, a major challenge in the identification of amusia is to set appropriate cutoffs. Although we argue, on the basis of the modular view of pitch processing, that cutoffs should rely on the melodic, rather than the temporal or memory, tests of the MBEA, some further methodological questions may be considered.

First, should a cutoff be applied to the average of the three melodic tests (melodic composite score), or should cutoffs be applied to each of the tests individually? A researcher's decision would depend on the goal of the study. For example, if one sought to identify amusics with the typical profile for a study on musical emotion, using a single cutoff for the melodic composite score would be appropriate. In contrast, if one were particularly interested in contour processing in amusics, applying cutoffs to each melodic test (especially the contour subtest) would be more useful.

A second important question is to consider how best to quantify performance on the MBEA subtests. Although accuracy has been the traditional method (Peretz \& Coltheart, 2003), recent critiques have proposed the use of $d$-prime to avoid the problem of overdiagnosis (Henry \& McAuley, 2010, 2012; Pfeifer \& Hamann, 2015). To address this issue, we compared accuracy and $d$-prime for a large sample of nonamusic participants whose full MBEA data had been collected at in our laboratory at BRAMS.
To provide a large, unbiased sample, we aggregated MBEA data from participants of the original MBEA validation study (Peretz et al., 2003) and a subsequent computerized MBEA validation study (unpublished). In total, the data set consisted of 175 participants (114 men, 61 women) 16 to 69 years of age $($ mean $=29.7)$ with 10 to 29 years of education (mean =15.7). As can be seen in Fig. 1, the distributions of accuracy and $d$-prime are significantly overlapping. As was reported by Henry and McAuley (2010), the accuracy distribution is more skewed to the left than the $d$-prime distribution, with skews $=-0.14$ and 0.06 , respectively. Both distributions are significantly nonnormal, Shapiro-Wilk $W=.91, p=.03$, and $W=.98, p=.01$, respectively.

Calculating a mean $-2 S D$ cutoff for each of the distributions gives an accuracy cutoff of 21.36 and a $d$-prime cutoff of 1.11. The application of these cutoffs to our sample of 175 participants led to the identification of eight potential amusics based on accuracy and five potential amusics based on $d$ prime. All five of the potential amusics identified using $d$ prime were in the group of eight identified using accuracy, which makes sense, given the high degree of overlap between the two distributions.

Whether to use accuracy or $d$-prime depends on the aim of the study. For instance, when estimating prevalence in a largescale study, $d$-prime might be useful to provide a more conservative estimate. However, when it comes to identifying amusic participants, the more liberal criterion provided by accuracy, in combination with the cognitive-control tasks described above, would be a more powerful approach.

\section{The amusic profile}

This final section constitutes a proof of concept of the MPIA. Here we compared a group of congenital amusics identified through this protocol (using the melodic composite score accuracy cutoff of 21.36 , calculated in the previous section) to a group of non-amusic control participants matched to the congenital amusics in terms of age, sex, and education (Table 2). These 13 amusics and 13 matched controls had been recruited over many years (2003 to present) to take part in studies in our laboratory. Three of these amusics and eight of the matched controls are from the sample $(n=175)$ used in the previous analyses. The remaining 164 participants were not tested with the full MPIA and were therefore excluded from the present analysis.

As is required by the MPIA, all of the participants had normal uncorrected age-standardized hearing, no history of traumatic brain injury, and performed within the normal range on all nonmusical cognitive screening tests. The amusics all had melodic composite scores below the cutoff. As expected, the controls outperformed the amusics on the scale test, $t(24)=$ $13.57, p<.001$, the off-beat test, $t(24)=3.48, p=.002$, and the 


\section{Melodic composite score}

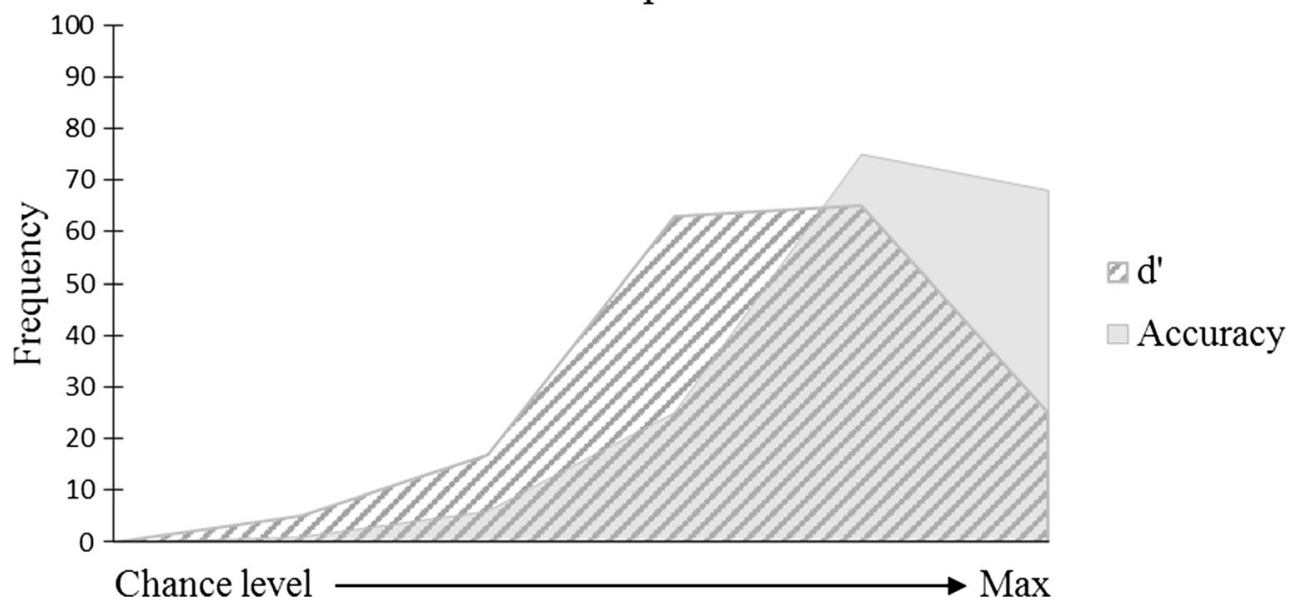

Fig. 1 Distribution of melodic composite scores from the MBEA ( $n=$ $175)$, presented in terms of both $d$-prime and accuracy (\% correct). For comparison, the $d$-prime and accuracy results have been superimposed by standardizing their distributions to have the same range (scores divided into six equal bins; $\min =$ chance level, $\max =$ maximum possible value)

controls as a group did not respond with a bias (mean melodic composite $C=-0.13$ ).

\section{Discussion}

It is our hope that the Montreal Protocol for Identification of Amusia will prove to be beneficial to both amusia researchers and the broader auditory cognitive-neuroscience community. As we have already discussed, the volume of research being conducted on amusia has been increasing consistently for a decade, due to its utility as an experimental model for auditory cognitive neuroscience. Thus, the standardization of the protocol to identify amusic participants will make it easier for results to be compared across studies and laboratories. This standardization will also lower the barrier to entry for groups who are new to this area of research.

The codification of the MPIA has provided us an opportunity to engage with the recent critiques of the MBEA (Henry \& McAuley, 2010, 2012; Pfeifer \& Hamann, 2015). These critiques have argued that the MBEA is overly liberal in identifying amusics when accuracy is used to quantify scores, that the cutoffs and subtests used to identify amusics are not stable across studies and research groups, and that online screening, employed in the AMUSIA tests, is less reliable than laboratory evaluation. As we have argued above, researchers should be able to decide whether to use accuracy versus $d$-prime, as well as which cutoffs and subtests to use, on the basis of the particular research question being asked. Furthermore, the combination of online and laboratory screening provides a more powerful and reliable method to identify amusics than does using either of those methods alone. Indeed, our protocol's approach of identifying amusic candidates by using liberal 
Table 3 MBEA performance for amusics $(n=13)$ and matched controls $(n=13)$

\begin{tabular}{|c|c|c|c|c|c|c|c|c|c|c|c|c|c|c|c|c|}
\hline \multirow[t]{2}{*}{ Group } & \multicolumn{2}{|l|}{ Scale } & \multicolumn{2}{|c|}{ Contour } & \multicolumn{2}{|c|}{ Interval } & \multicolumn{2}{|c|}{ Rhythm } & \multicolumn{2}{|c|}{ Meter } & \multicolumn{2}{|c|}{ Memory } & \multicolumn{2}{|c|}{ Melodic } & \multicolumn{2}{|c|}{ Global } \\
\hline & acc & $d^{\prime}$ & acc & $d^{\prime}$ & acc & $d^{\prime}$ & acc & $d^{\prime}$ & acc & $d^{\prime}$ & acc & $d^{\prime}$ & acc & $d^{\prime}$ & acc & $d^{\prime}$ \\
\hline Amusics & $\begin{array}{l}18.3 \\
(3.2)\end{array}$ & $\begin{array}{l}0.7 \\
(0.9)\end{array}$ & $\begin{array}{l}19.1 \\
(2.5)\end{array}$ & $\begin{array}{l}0.9 \\
(0.6)\end{array}$ & $\begin{array}{l}18.1 \\
(2.8)\end{array}$ & $\begin{array}{l}0.7 \\
(0.6)\end{array}$ & $\begin{array}{l}22.1 \\
(3.5)\end{array}$ & $\begin{array}{l}1.6 \\
(0.7)\end{array}$ & $\begin{array}{l}19.5 \\
(3.9)\end{array}$ & $\begin{array}{l}0.9 \\
(0.9)\end{array}$ & $\begin{array}{l}21.5 \\
(3.4)\end{array}$ & $\begin{array}{l}1.4 \\
(0.6)\end{array}$ & $\begin{array}{l}18.5 \\
(2.0)\end{array}$ & $\begin{array}{l}0.8 \\
(0.5)\end{array}$ & $\begin{array}{l}19.8 \\
(1.8)\end{array}$ & $\begin{array}{l}1.1 \\
(0.4)\end{array}$ \\
\hline Controls & $\begin{array}{l}27.8 \\
(1.8)\end{array}$ & $\begin{array}{l}3.1 \\
(0.7)\end{array}$ & $\begin{array}{l}27.2 \\
(1.8)\end{array}$ & $\begin{array}{l}2.8 \\
(0.8)\end{array}$ & $\begin{array}{l}26.9 \\
(2.5)\end{array}$ & $\begin{array}{l}2.9 \\
(0.8)\end{array}$ & $\begin{array}{l}27.5 \\
(1.9)\end{array}$ & $\begin{array}{l}3.2 \\
(0.7)\end{array}$ & $\begin{array}{l}27.7 \\
(2.5)\end{array}$ & $\begin{array}{l}3.2 \\
(1.1)\end{array}$ & $\begin{array}{l}27.5 \\
(2.3)\end{array}$ & $\begin{array}{l}3.1 \\
(0.9)\end{array}$ & $\begin{array}{l}27.3 \\
(1.6)\end{array}$ & $\begin{array}{l}3.0 \\
(0.6)\end{array}$ & $\begin{array}{l}27.4 \\
(1.3)\end{array}$ & $\begin{array}{l}3.1 \\
(0.5)\end{array}$ \\
\hline
\end{tabular}

acc $=$ accuracy $(\%$ correct $), d^{\prime}=d$-prime. The numbers appearing in parentheses are standard errors. Comparisons between amusics and controls were significant, $p<.001$, for all six subtests.

screening criteria with the MBEA, followed by a more conservative examination of these potential cases with further control testing, has a long history in medical diagnostics (Garland, 1949).

In sum, the MPIA's combination of music-specific and cognitive-control tasks, administered both online and in the laboratory, provides researchers with a standardized protocol for identifying amusics that is transparent, empirically grounded, and flexible to the needs of researchers in varying contexts. The latter attribute is most important, because scientific standards should be flexible, so as to incorporate new knowledge into current practice. The protocol for identifying amusics is therefore expected to evolve over time as researchers continue to illuminate our understanding of this disorder.

For example, a decade of neuroscientific research has offered promising leads on a diagnostic neurofunctional marker for amusia. Electroencephalographic work has indicated that amusics generally have intact early responses to pitch, as indexed by the mismatch negativity or $\mathrm{N} 2$, but disturbed later responses, as indexed by the P3, P300, or P600 (Mignault Goulet et al., 2012; Moreau, Jolicœur, \& Peretz, 2013; Zendel, Lagrois, Robitaille, \& Peretz, 2015). This neurophysiological anomaly is accompanied by anatomical and functional anomalies along the right fronto-temporal pathway (Hyde et al., 2007; Hyde, Zatorre, Griffiths, Lerch, \& Peretz, 2006; Hyde, Zatorre, \& Peretz, 2011; Loui et al., 2009; but see Chen et al., 2015). "Reverse-engineering" studies that have used brain stimulation methods to elicit the amusic phenotype in nonamusic participants offer further evidence of an abnormal right fronto-temporal pathway as a neurological marker for amusia (Loui, Hohmann, \& Schlaug, 2010; Mathys, Loui, Zheng, \& Schlaug, 2010).

At present, these neurophysiological markers have been seen at the group level, rather than being individually diagnostic, but as the precision of neuroimaging methods increases, we may be able to move toward the individual identification of amusic brains. One such potentially useful technique is multivoxel pattern analysis, which compares distributed, rather than focal, patterns of activation, and is therefore more sensitive to group differences (e.g., amusics vs. controls) than is traditional univariate analysis.

Another challenge inherent in identifying amusics has come from evidence for phenotypic heterogeneity within congenital amusia on a variety of dimensions (see Vuvan et al., 2015, for a discussion). A subset of amusics is able to produce pitches accurately despite a pitch perception deficit (Hutchins $\&$ Peretz, 2012). Additionally, Nan and colleagues have identified tone-language-speaking amusics who do and do not have difficulties with lexical tone perception (Huang, Nan, Dong, \& Liu, 2015; Nan, Sun, \& Peretz, 2010). Amusics vary widely in their music interests: Many feel indifferent, some report irritation, and a minority are music lovers (Ayotte, Peretz, \& Hyde, 2002; Falconer, 2016; Gosselin et al., 2015; McDonald \& Stewart, 2008; Omigie, Pearce, Williamson, \& Stewart (2013); Wilbiks, Vuvan, Girard, Peretz, \& Russo, 2016). In contrast, most amusics can, despite their deficit, correctly identify the emotions conveyed in music (Gosselin et al., 2015). Recently, the predicted dissociation between melodic and rhythmic abilities has led to the identification of beat deafness as a distinct disorder that is characterized by impairments in the temporal domain, rather than the pitch domain upon which we are presently focused (Phillips-Silver et al., 2011; Tranchant, Vuvan, \& Peretz, 2016). Another line of research has begun to explore amusia in a developmental context (Lebrun, Moreau, McNally-Gagnon, Mignault Goulet, \& Peretz, 2012; Mignault Goulet et al., 2012), and a new tool has been developed to identify amusia in children: The Montreal Battery of Evaluation of Musical Abilities (MBEMA; Peretz et al., 2013). Thus, it is clear that as our understanding of amusia expands, the protocol through which we identify amusics will expand as well.

It is important to note that the question of how best to identify individuals with amusia is far from unique in the field of neuropsychology. Researchers studying such disorders as dyslexia and specific language impairment (e.g., Aram, Morris, \& Hall, 1993; Bishop \& Snowling, 2004; Tomblin, Records, \& Zhang, 1996), dyscalculia (e.g., Butterworth \& Laurillard, 2010; Shalev, Auerbach, Manor, \& Gross-Tsur, 2000), and prosopagnosia (e.g., Bowles et al., 2009; 
Duchaine \& Nakayama, 2006; Towler, Fisher, \& Eimer, 2017) face the same challenge. For these disorders, which all lack a single, unambiguous defining characteristic such as a specific genotype, individual performance must be categorized as "impaired" on the basis of continuous distributions of scores, meaning that the cutoff is largely arbitrary and is developed via consensus in the field (Towler et al., 2017). As was argued by Henry and McAuley (2010), one way to develop a more rigorous practice around the identification of these disorders will be to use multiple dependent measures and to compare patterns of performance to theoretically defined control versus disordered phenotypes. The protocol presented here constitutes a starting point for implementing this approach for amusia.

As such, we believe that it is important to provide the materials that constitute the MPIA for use and discussion by the larger research community. To this end, a participant scoring sheet for the MPIA, MBEA materials, and online AMUSIA test materials are available for download at www. peretzlab.ca/knowledge_transfer/. We hope that the codification of the MPIA and our open provision of these materials will help stimulate collaborative research within an already very active and productive scientific community.

Author note The authors thank Nathalie Gosselin and Marina Dallongeville for their help with data collection, Joël Paquette and Marielle Saucier for their help with data processing, Peer Herholz for his work digitizing the MBEA, and our dedicated participants, without whom this research would not have been possible. This research was financially supported by a grant from the Canada Research Chair program to I.P., a grant from the Natural Sciences and Engineering Research Council of Canada to I.P., a grant from Fonds de Recherche Nature et Technologies Quebec to D.T.V., and grants from the Canadian Institutes of Health Research to I.P., S.P., and G.M.G.

\section{References}

Albouy, P., Mattout, J., Bouet, R., Maby, E., Sanchez, G., Aguera, P.-E., . . . Caclin, A. (2013). Impaired pitch perception and memory in congenital amusia: The deficit starts in the auditory cortex. Brain, 136, 1639-1661. doi:10.1093/brain/awt082

Albouy, P., Schulze, K., Caclin, A., \& Tillmann, B. (2013). Does tonality boost short-term memory in congenital amusia? Brain Research, 1537, 224-232. doi:10.1016/j.brainres.2013.09.003

Anderson, S., Himonides, E., Wise, K., Welch, G., \& Stewart, L. (2012). Is there potential for learning in amusia? A study of the effect of singing intervention in congenital amusia. Annals of the New York Academy of Sciences, 1252, 345-353. doi:10.1111/j.1749-6632. 2011.06404.x

Aram, D. M., Morris, R., \& Hall, N. E. (1993). Clinical and research congruence in identifying children with specific language impairment. Journal of Speech, Language, and Hearing Research, 36, 580-591. doi:10.1044/jshr.3603.580

Ayotte, J., Peretz, I., \& Hyde, K. (2002). Congenital amusia: A group study of adults afflicted with a music-specific disorder. Brain, 125, 238-251. doi:10.1093/brain/awf028
Bishop, D. V., \& Snowling, M. J. (2004). Developmental dyslexia and specific language impairment: Same or different? Psychological Bulletin, 130, 858-886. doi:10.1037/0033-2909.130.6.858

Bowles, D. C., McKone, E., Dawel, A., Duchaine, B., Palermo, R., Schmalzl, L., . . Y Yovel, G. (2009). Diagnosing prosopagnosia: Effects of ageing, sex, and participant-stimulus ethnic match on the Cambridge Face Memory Test and Cambridge Face Perception Test. Cognitive Neuropsychology, 26, 423-455. doi:10.1080/ 02643290903343149

Butterworth, B., \& Laurillard, D. (2010). Low numeracy and dyscalculia: Identification and intervention. ZDM Mathematics Education, 42, 527-539. doi:10.1007/s11858-010-0267-4

Chen, J. L., Kumar, S., Williamson, V. J., Scholz, J., Griffiths, T. D., \& Stewart, L. (2015). Detection of the arcuate fasciculus in congenital amusia depends on the tractography algorithm. Frontiers in Psychology, 6, 9. doi:10.3389/fpsyg.2015.00009

Dalla Bella, S., Giguère, J.-F., \& Peretz, I. (2009). Singing in congenital amusia. Journal of the Acoustical Society of America, 126, 414- 424. doi:10.1121/1.3132504

Duchaine, B., \& Nakayama, K. (2006). The Cambridge Face Memory Test: Results for neurologically intact individuals and an investigation of its validity using inverted face stimuli and prosopagnosic participants. Neuropsychologia, 44, 576-585. doi:10.1016/j. neuropsychologia.2005.07.001

Falconer, T. (2016). Bad Singer: The Surprising Science of Tone Deafness and How We Hear Music. Toronto, ON: House of Anansi Press, Inc.

Foxton, J. M., Dean, J. L., Gee, R., Peretz, I., \& Griffiths, T. D. (2004). Characterization of deficits in pitch perception underlying 'tone deafness'. Brain, 127, 801-810. doi:10.1093/brain/awh105

Garland, L. H. (1949). On the scientific evaluation of diagnostic procedures: Presidential address at the Thirty-Fourth Annual Meeting of the Radiological Society of North America. Radiology, 52, 309328. doi:10.1148/52.3.309

Gosselin, N., Jolicœur, P., \& Peretz, I. (2009). Impaired memory for pitch in congenital amusia. Annals of the New York Academy of Sciences, 1169, 270-272. doi:10.1111/j.1749-6632.2009.04762.x

Gosselin, N., Paquette, S., \& Peretz, I. (2015). Sensitivity to musical emotions in congenital amusia. Cortex, 71, 171-182. doi:10.1111/ j.1749-6632.2009.04762.x

Henry, M. J., \& McAuley, J. D. (2010). On the prevalence of congenital amusia. Music Perception, 27, 413-418. doi:10.1525/mp.2010.27. 5.413

Henry, M. J., \& McAuley, J. D. (2012). Failure to apply signal detection theory to the Montreal Battery of Evaluation of Amusia may misdiagnose amusia. Music Perception, 30, 480-496. doi:10.1525/mp. 2013.30.5.480

Huang, W.-T., Nan, Y., Dong, Q., \& Liu, C. (2015). Just-noticeable difference of tone pitch contour change for Mandarin congenital amusics. The Journal of the Acoustical Society of America, 138, EL99-EL104. doi:10.1121/1.4923268

Hutchins, S., Gosselin, N., \& Peretz, I. (2010). Identification of changes along a continuum of speech intonation is impaired in congenital amusia. Frontiers in Psychology, 1, 236. doi:10.3389/fpsyg.2010. 00236

Hutchins, S., \& Peretz, I. (2012). Amusics can imitate what they cannot discriminate. Brain and Language, 123, 234-239. doi:10.3389/ fpsyg.2010.00236

Hutchins, S., \& Peretz, I. (2013). Vocal pitch shift in congenital amusia (pitch deafness). Brain and Language, 125, 106-117. doi:10.1016/j. bandl.2013.01.011

Hutchins, S., Zarate, J. M., Zatorre, R. J., \& Peretz, I. (2010). An acoustical study of vocal pitch matching in congenital amusia. The Journal of the Acoustical Society of America, 127, 504-512. doi: 10.1121/1.3270391

Hyde, K. L., Lerch, J. P., Zatorre, R. J., Griffiths, T. D., Evans, A. C., \& Peretz, I. (2007). Cortical thickness in congenital amusia: When less 
is better than more. The Journal of Neuroscience, 27, 13028-13032. doi:10.1523/JNEUROSCI.3039-07.2007

Hyde, K. L., \& Peretz, I. (2004). Brains that are out of tune but in time. Psychological Science, 15, 356-360. doi:10.1111/j.0956-7976. 2004.00683.x

Hyde, K. L., \& Peretz, I. (2005). Congenital amusia: Impaired musical pitch but intact musical time. In J. Syka \& M. M. Merzenich (Eds.), Plasticity and signal representation in the auditory system. New York: Springer.

Hyde, K. L., Zatorre, R. J., Griffiths, T. D., Lerch, J. P., \& Peretz, I. (2006). Morphometry of the amusic brain: A two-site study. Brain, 129, 2562-2570. doi:10.1093/brain/awl204

Hyde, K. L., Zatorre, R. J., \& Peretz, I. (2011). Functional MRI evidence of an abnormal neural network for pitch processing in congenital amusia. Cerebral Cortex, 21, 292-299. doi:10.1093/cercor/bhq094

Jiang, C., Hamm, J. P., Lim, V. K., Kirk, I. J., Chen, X., \& Yang, Y. (2012). Amusia results in abnormal brain activity following inappropriate intonation during speech comprehension. PLoS ONE, 7, e41411. doi:10.1371/journal.pone.0041411

Jiang, C., Hamm, J. P., Lim, V. K., Kirk, I. J., \& Yang, Y. (2010). Processing melodic contour and speech intonation in congenital amusics with Mandarin Chinese. Neuropsychologia, 48, 2630 2639. doi:10.1371/journal.pone.0041411

Jiang, C., Hamm, J. P., Lim, V. K., Kirk, I. J., \& Yang, Y. (2011). Finegrained pitch discrimination in congenital amusics with Mandarin Chinese. Music Perception, 28, 519-526. doi:10.1525/mp.2011.28. 5.519

Jiang, C., Lim, V. K., Wang, H., \& Hamm, J. P. (2013). Difficulties with pitch discrimination influences pitch memory performance: Evidence from congenital amusia. PLoS ONE, 8, e79216. doi:10. 1371/journal.pone.0079216

Lebrun, M.-A., Moreau, P., McNally-Gagnon, A., Mignault Goulet, G., \& Peretz, I. (2012). Congenital amusia in childhood: a case study. Cortex, 48, 683-688. doi:10.1016/j.cortex.2011.02.018

Liu, F., Jiang, C., Thompson, W. F., Xu, Y., Yang, Y., \& Stewart, L. (2012). The mechanism of speech processing in congenital amusia: Evidence from Mandarin speakers. PLoS ONE, 7, e30374. doi:10. 1371/journal.pone.0030374

Liu, F., Patel, A. D., Fourcin, A., \& Stewart, L. (2010). Intonation processing in congenital amusia: Discrimination, identification and imitation. Brain, 133, 1682-1693. doi:10.1093/brain/awq089

Liu, F., Xu, Y., Patel, A. D., Francart, T., \& Jiang, C. (2012). Differential recognition of pitch patterns in discrete and gliding stimuli in congenital amusia: Evidence from Mandarin speakers. Brain and Cognition, 79, 209-215. doi:10.1016/j.bandc.2012.03.008

Loui, P., Alsop, D., \& Schlaug, G. (2009). Tone deafness: A new disconnection syndrome? The Journal of Neuroscience, 29, 10215-10220. doi:10.1523/JNEUROSCI.1701-09.2009

Loui, P., Guenther, F. H., Mathys, C., \& Schlaug, G. (2008). Actionperception mismatch in tone-deafness. Current Biology, 18, R331R332. doi:10.1016/j.cub.2008.02.045

Loui, P., Hohmann, A., \& Schlaug, G. (2010). Inducing disorders in pitch perception and production: A reverse-engineering approach. Proceedings of Meetings on Acoustics, 9, 050002. doi:10.1121/1. 3431713

Loui, P., \& Schlaug, G. (2012). Impaired learning of event frequencies in tone deafness. Annals of the New York Academy of Sciences, 1252, 354-360. doi:10.1111/j.1749-6632.2011.06401.x

Marin, M. M., Gingras, B., \& Stewart, L. (2012). Perception of musical timbre in congenital amusia: Categorization, discrimination and short-term memory. Neuropsychologia, 50, 367-378. doi:10.1016/ j.neuropsychologia.2011.12.006

Marin, M. M., Thompson, W. F., Gingras, B., \& Stewart, L. (2015). Affective evaluation of simultaneous tone combinations in congenital amusia. Neuropsychologia, 78, 207-220. doi:10.1016/j. neuropsychologia.2015.10.004
Mathys, C., Loui, P., Zheng, X., \& Schlaug, G. (2010). Non-invasive brain stimulation applied to Heschl's gyrus modulates pitch discrimination. Frontiers in Psychology, 1, 193. doi:10.3389/fpsyg.2010. 00193

McDonald, C., \& Stewart, L. (2008). Uses and functions of music in congenital amusia. Music Perception, 25, 345-355. doi:10.1525/ mp.2008.25.4.345

Mignault Goulet, G., Moreau, P., Robitaille, N., \& Peretz, I. (2012). Congenital amusia persists in the developing brain after daily music listening. PLoS ONE, 7, e36860. doi:10.1371/journal.pone.0036860

Moreau, P., Jolicœur, P., \& Peretz, I. (2013). Pitch discrimination without awareness in congenital amusia: evidence from event-related potentials. Brain and Cognition, 81, 337-344. doi:10.1371/journal.pone. 0036860

Nan, Y., Sun, Y., \& Peretz, I. (2010). Congenital amusia in speakers of a tone language: association with lexical tone agnosia. Brain, 133 2635-2642. doi:10.1093/brain/awq178

Nasreddine, Z. S., Phillips, N. A., Bédirian, V., Charbonneau, S., Whitehead, V., Collin, I., . . Chertkow, H. (2005). The Montreal Cognitive Assessment, MoCA: A brief screening tool for mild cognitive impairment. Journal of the American Geriatrics Society, 53, 695-699. doi:10.1111/j.1532-5415.2005.53221.x

Omigie, D., Müllensiefen, D., \& Stewart, L. (2012). The experience of music in congenital amusia. Music Perception, 30, 1-18. doi:10. 1525/mp.2012.30.1.1

Omigie, D., Pearce, M. T., Williamson, V. J., \& Stewart, L. (2013). Electrophysiological correlates of melodic processing in congenital amusia. Neuropsychologia, 51, 1749-1762. doi:10.1016/j. neuropsychologia.2013.05.010

Omigie, D., Pearce, M. T., \& Stewart, L. (2012). Tracking of pitch probabilities in congenital amusia. Neuropsychologia, 50, 1483-1493. doi:10.1016/j.neuropsychologia.2012.02.034

Omigie, D., \& Stewart, L. (2011). Preserved statistical learning of tonal and linguistic material in congenital amusia. Frontiers in Psychology, 2, 1-11. doi:10.3389/fpsyg.2011.00109

Patel, A. D., Wong, M., Foxton, J., Lochy, A., \& Peretz, I. (2008). Speech intonation perception deficits in musical tone deafness (congenital amusia). Music Perception, 25, 357-368. doi:10.1525/mp.2008.25. 4.357

Peretz, I. (2016). Neurobiology of Congenital Amusia. Trends in Cognitive Sciences, 20, 857-867. doi:10.1016/j.tics.2016.09.002

Peretz, I., Brattico, E., Järvenpää, M., \& Tervaniemi, M. (2009). The amusic brain: In tune, out of key, and unaware. Brain, 132, 12771286. doi:10.1093/brain/awp055

Peretz, I., Champod, A. S., \& Hyde, K. (2003). Varieties of musical disorders. Annals of the New York Academy of Sciences, 999, 58 75. doi:10.1196/annals. 1284.006

Peretz, I., \& Coltheart, M. (2003). Modularity of music processing. Nature Neuroscience, 6, 688-691. doi:10.1038/nn1083

Peretz, I., Cummings, S., \& Dubé, M.-P. (2007). The genetics of congenital amusia (tone deafness): a family aggregation study. The American Journal of Human Genetics, 81, 582-588. doi:10.1086/ 521337

Peretz, I., Gosselin, N., Nan, Y., Caron-Caplette, E., Trehub, S. E., \& Béland, R. (2013). A novel tool for evaluating children's musical abilities across age and culture. Frontiers in Systems Neuroscience, 7, 30. doi:10.3389/fnsys.2013.00030

Peretz, I., Gosselin, N., Tillmann, B., Cuddy, L. L., Gagnon, B., Trimmer, C. G., .. . Bouchard, B. (2008). On-line identification of congenital amusia. Music Perception, 25, 331-343. doi:10.1525/mp.2008.25. 4.331

Peretz, I., \& Vuvan, D. T. (2017). Prevalence of congenital amusia. European Journal of Human Genetics. doi:10.1038/ejhg.2017.15

Pfeifer, J., \& Hamann, S. (2015). Revising the diagnosis of congenital amusia with the Montreal Battery of Evaluation of Amusia. 
Frontiers in Human Neuroscience, 9, 161. doi:10.3389/fnhum. 2015.00161

Pfeuty, M., \& Peretz, I. (2010). Abnormal pitch-time interference in congenital amusia: Evidence from an implicit test. Attention, Perception, \& Psychophysics, 72, 763-774. doi:10.3758/APP.72.3. 763

Phillips-Silver, J., Toiviainen, P., Gosselin, N., Piché, O., Nozaradan, S., Palmer, C., \& Peretz, I. (2011). Born to dance but beat deaf: A new form of congenital amusia. Neuropsychologia, 49, 961-969. doi:10. 1016/j.neuropsychologia.2011.02.002

Phillips-Silver, J., Toiviainen, P., Gosselin, N., \& Peretz, I. (2013). Amusic does not mean unmusical: Beat perception and synchronization ability despite pitch deafness. Cognitive Neuropsychology, 30, 311-331. doi:10.1080/02643294.2013.863183

Royal, I., Lidji, P., Théoret, H., Russo, F. A., \& Peretz, I. (2015). Excitability of the motor system: A transcranial magnetic stimulation study on singing and speaking. Neuropsychologia, 75, 525532. doi:10.3758/APP.72.3.763

Shalev, R. S., Auerbach, J., Manor, O., \& Gross-Tsur, V. (2000). Developmental dyscalculia: Prevalence and prognosis. European Child \& Adolescent Psychiatry, 9, S58-S64. doi:10.1007/ s007870070009

Thompson, W. F., Marin, M. M., \& Stewart, L. (2012). Reduced sensitivity to emotional prosody in congenital amusia rekindles the musical protolanguage hypothesis. Proceedings of the National Academy of Sciences, 109, 19027-19032. doi:10.1073/pnas. 1210344109

Tillmann, B., Burnham, D., Nguyen, S., Grimault, N., Gosselin, N., \& Peretz, I. (2011). Congenital amusia (or tone-deafness) interferes with pitch processing in tone languages. Frontiers in Psychology, 2. doi:10.3389/fpsyg.2011.00120

Tillmann, B., Rusconi, E., Traube, C., Butterworth, B., Umiltà, C., \& Peretz, I. (2011). Fine-grained pitch processing of music and speech in congenital amusia. The Journal of the Acoustical Society of America, 130, 4089-4096. doi:10.1121/1.3658447

Tillmann, B., Schulze, K., \& Foxton, J. M. (2009). Congenital amusia: A short-term memory deficit for non-verbal, but not verbal sounds. Brain and Cognition, 71, 259-264. doi:10.1016/j.bandc.2009.08. 003

Tomblin, J. B., Records, N. L., \& Zhang, X. (1996). A system for the diagnosis of specific language impairment in kindergarten children. Journal of Speech, Language, and Hearing Research, 39, 1284 1294. doi:10.1044/jshr.3906.1284
Towler, J., Fisher, K., \& Eimer, M. (2017). The cognitive and neural basis of developmental prosopagnosia. The Quarterly Journal of Experimental Psychology, 70, 316-344. doi:10.1080/17470218. 2016.1165263

Tranchant, P., Vuvan, D. T., \& Peretz, I. (2016). Keeping the beat: A large sample study of bouncing and clapping to music. PLOS ONE, 11, e0160178. doi:10.1371/journal.pone.0160178

Tremblay-Champoux, A., Dalla Bella, S., Phillips-Silver, J., Lebrun, M.A., \& Peretz, I. (2010). Singing proficiency in congenital amusia: Imitation helps. Cognitive Neuropsychology, 27, 463-476. doi:10. 1080/02643294.2011.567258

Tulsky, D., Zhu, J., \& Ledbetter, M. (1997). Wechsler Adult Intelligence Scale and Wechsler Memory Scale technical manual. San Antonio, TX: Psychological Corp.

Vuvan, D. T., Nunes-Silva, M., \& Peretz, I. (2015). Meta-analytic evidence for the non-modularity of pitch processing in congenital amusia. Cortex, 69, 186-200. doi:10.1016/j.cortex.2015.05.002

Wechsler, D. (1997). WAIS-III: Wechsler adult intelligence scale. San Antonio, TX: Psychological Corp.

Wilbiks, J. M., Vuvan, D. T., Girard, P.-Y., Peretz, I., \& Russo, F. A. (2016). Effects of vocal training in a musicophile with congenital amusia. Neurocase, 22, 526-537. doi:10.1080/13554794.2016. 1263339

Williamson, V. J., Cocchini, G., \& Stewart, L. (2011). The relationship between pitch and space in congenital amusia. Brain and Cognition, 76. 70-76. doi:10.1016/j.bandc.2011.02.016

Williamson, V. J., Liu, F., Peryer, G., Grierson, M., \& Stewart, L. (2012). Perception and action de-coupling in congenital amusia: Sensitivity to task demands. Neuropsychologia, 50, 172-180. doi:10.1016/j. neuropsychologia.2011.11.015

Williamson, V. J., McDonald, C., Deutsch, D., Griffiths, T. D., \& Stewart, L. (2010). Faster decline of pitch memory over time in congenital amusia. Advances in Cognitive Psychology, 6, 15-22. doi:10.2478/ v10053-008-0073-5

Williamson, V. J., \& Stewart, L. (2010). Memory for pitch in congenital amusia: Beyond a fine-grained pitch discrimination problem. Memory, 18, 657-669. doi:10.1080/09658211.2010.501339

Zendel, B. R., Lagrois, M.-É., Robitaille, N., \& Peretz, I. (2015). Attending to pitch information inhibits processing of pitch information: The curious case of amusia. The Journal of Neuroscience, 35, 3815-3824. doi:10.1523/JNEUROSCI.3766-14.2015 